\title{
Unusual variant of type 3 dens invaginatus in a maxillary lateral: A rare case report
}

\author{
Neslihan Yılmaz Çırakoğlu, ${ }^{1}$ (i) Ersan Çiçek² \\ 'Department of Endodontics, Karabük University Faculty of Dentistry, Karabük, Turkey \\ 2Private Dentist, Samsun, Turkey
}

\begin{abstract}
Dens invaginatus is a developmental anomaly resulting from epithelial invagination into the dental papilla. The etiology of the dens invaginatus malformation remains undefined. The most commonly affected tooth is the maxillary lateral incisor followed by the maxillary central incisor. Endodontic treatment of teeth with dens invaginatus can be highly complicated due to anatomical challenges. Treatment options include nonsurgical treatment of the main canal and invagination, endodontic surgery, a combination of these techniques, intentional replantation and extraction. This case report presents the successful nonsurgical endodontic treatment of a maxillary right lateral diagnosed with an interesting type III dens invaginatus and communicated extensive periapical lesion. The results obtained after five years of clinical and radiographic follow-up demonstrated that conventional endodontic treatment is a clinically viable alternative in cases of type III dens invaginatus.
\end{abstract}

Keywords: Cone beam computed tomography; dens invaginatus; immature apex; nonsurgical endodontic treatment.

$\mathrm{D}$ ens invaginatus is a developmental anomaly resulting from epithelial invagination into the dental papilla.

${ }^{[1]}$ The prevalence of dens invaginatus in teeth is between $0.4 \%$ and $10 \%$. The most commonly affected tooth is the maxillary lateral incisor followed by the maxillary central incisor. Other teeth, including the maxillary canine, are rarely invaginated. Bilateral occurrence is not an uncommon finding. ${ }^{[2,3]}$

The etiology of the dens invaginatus malformation remains undefinite. Many factors have been suggested to describe this unusual dental formation, including, disruption of factor that coordinate the formation of the enamel organ,inhibition of the growth of spesific cells, infection, trauma and links to genetic factors. ${ }^{[1,3,4]}$
Oehlers ${ }^{[5]}$ was the first to define III different types of dens invaginatus, that were classified according to the depth of the invagination into the root. Type I invaginations remain confined within the crown. In Type II invagination, there is extension of the invagination apical to the cementoenamel junction ending as a blind pouch within the root canal system but not reaching the periodontal ligament. Type III is the form in which invagination penetrates through the root, perforating at the apical area and showing a second foramen in the apical or in the periodontal area. In type III lesions, any connection between the oral cavity and the invaginatus foramen may cause to an inflammatory reply within the periodontal tissues. The disease is characterized by peri-invagination periodontitis

Correspondence: Dr. Neslihan Yılmaz Çırakoğlu. Karabük Üniversitesi Diş Hekimliği Fakültesi,

Endodonti Anabilim Dalı, Karabük, Turkey

Tel: +90 505 - 3006401 e-mail: neslihanyilmazcirakoglu@karabuk.edu.tr

Submitted: March 19, 2020 Accepted: May 01, 2020

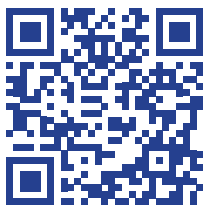


by some authors. ${ }^{[1]}$ Also invagination permits access of microorganisms into pulp space through a thin hypo-mineralised enamel and dentin stratum. This microorganism penetration leads to pulp necrosis,cyst and abscess formation, tooth displacement and internal resorption. ${ }^{[6]}$ Therefore, it requires the cleaning and sealing of the invagination area to support healing.

Endodontic treatment of teeth with dens invaginatus can be highly complicated due to anatomical challenges. Treatment options include nonsurgical treatment of the main canal and invagination, endodontic surgery, a combination of these techniques, intentional replantation and extraction. ${ }^{[7]}$

This article reports the successful nonsurgical endodontic treatment of a maxillary right lateral diagnosed with type III dens invaginatus and communicated periapical lesion.

\section{Case report}

A 25-year-old female patient was referred to the Department of Endodontics because of pain and swelling arising from the maxillary right lateral incisor (Fig. la). The patient's medical history was noncontributory. The oral hygiene condition was moderate; the intraoral soft tissues were free of pathological signs expect in the labial area of the maxillary right lateral incisor, where a clear swelling was noticed. The lateral tooth has a unusual morphologic form, the clinical crown especially the mesiodistal diameter was larger compared to the lateral incisor on the left side. The tooth was caries-free and had no restoration in it (Fig. la and b). The tooth was tender on vertical percussion. There were no horizontal mobility and depressibility. Also the canine tooth was not available. The tooth did not respond to electric pulp vitality test with an electric pulp tester (Electric Pulp Tester; Parkell, Farmingdale, NY, USA) and cold tests, however adjacent teeth all responded within normal limits. A periapikal radiograph of the area exhibited dens invaginatus of the right lateral incisor. Radiographic examination revealed an invagination extending to the root, and communicating with the periodontal ligament with an open-foramen and a large periapical lesion (Fig. 2a). Moreover, cone beam computed tomography was taken to determine the exact diagnosis and dimension of the periapical lesion (Fig. 3). Under local anesthesia, rubber dam was applied on the tooth and endodontic access was performed with round bur. By using an endodontic explorer and a size $10 \mathrm{~K}$-file, 2 orifices were identified (Fig. 1c). The canals were negotiated and working lengths was determined with an electronic apex locator (Root ZX mini; J Morita Co, Kyoto, Japan) and radiograph. The main root canal was instrumented with ProTaper (Dentsply Maillefer, Ballaigues, Switzerland) instruments up to F3. The space of invagination was instrumented with K-files (Dentsply Maillefer, Ballaigues, Switzerland). During the instrumentation, the main canal and invaginated space were irrigated with copious amounts of $2.5 \%$ sodium hypochlorite and $17 \%$ EDTA. The agitation of the irrigant was done by using ultrasonics (Endo tips; Satelec Ultrasonic Scaler, Bordeaux, France) and dried with sterile absorbent paper points. After the final irrigation the canals were dressed with calcium hydroxide paste for 2 weeks (RC Cal; Prime Dental Products, Mumbai, India), and the access was sealed with temporary filling material $(\mathrm{Ca}-$ vit G;3M ESPE, Germany).

At the second appointment the tooth was asymptomatic and the sinus tract was closed (Fig. 1d). After removal of calcium hydroxide with $2.5 \%$ sodium hypochlorite. After the final irrigation, final obturation of the main root canal was performed with gutta-percha and AHplus sealer ((Dentsply, De Trey, GmbH, Konstanz, Germany) by using the cold lateral condensation technique. Apexification and complete obturation of the invaginated canal were done with white mineral trioxide aggregate (MTA) (Angelus Dental Solutions, Londrina, PR, Brazil) (Fig. 2b). Moist cotton plug was placed over MTA, the access was sealed with Cavit, and patient was recalled after 24 hours. The tooth was restored finally with resin modified glass
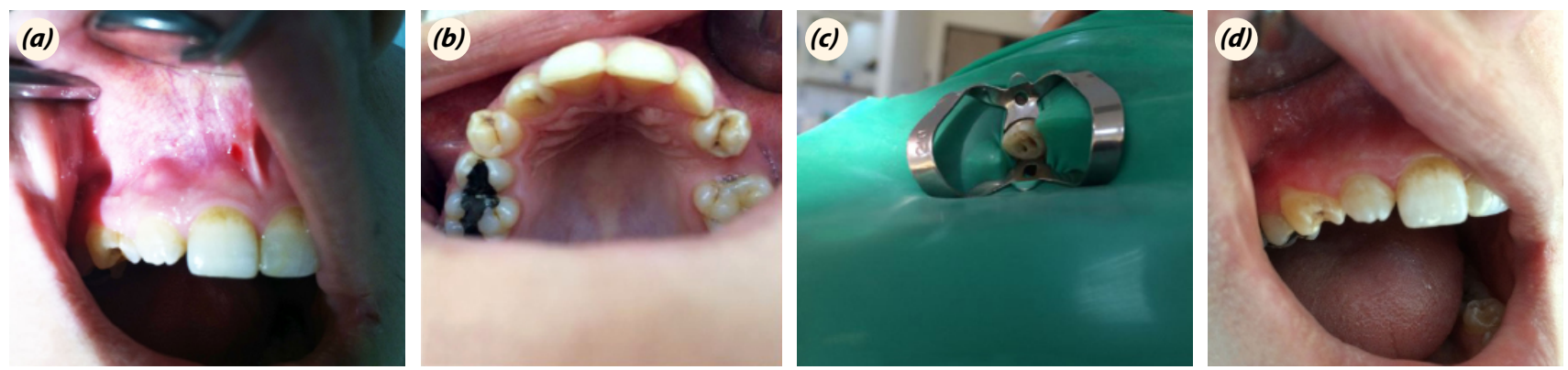

Fig. 1. (a) Preoperative clinical appearances of the invaginated tooth and buccal mucosa. (b) Preoperative occlusal appearance of the invaginated tooth. (c) Clinical appearance of canal orificies. (d) Intraoral clinical appearance after $\mathrm{Ca}(\mathrm{OH})_{2}$ dressing. 

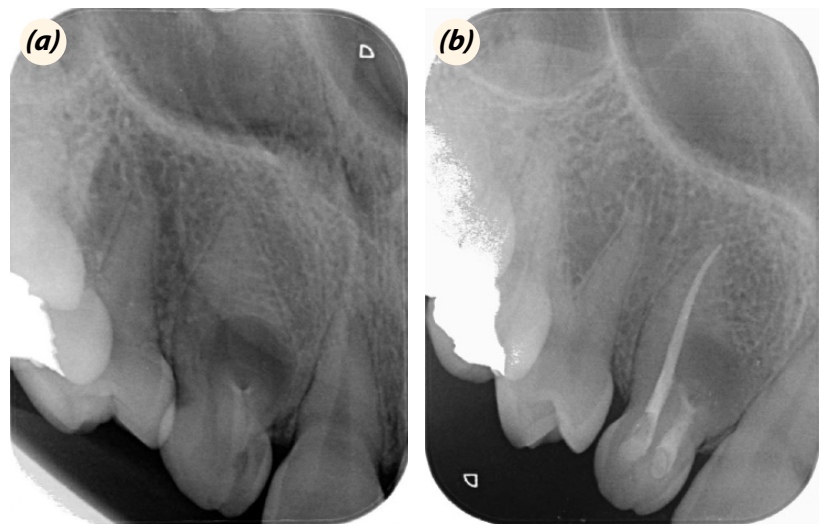

Fig. 2. (a) Periapical radiographic appearance of dens invaginatus. (b) Postoperative radiographic appearance after root canal filling.

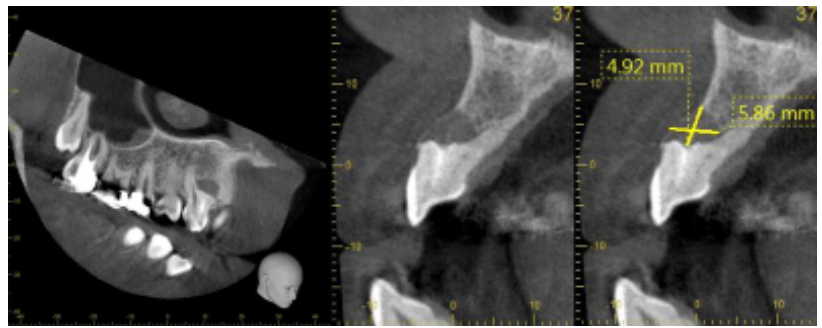

Fig. 3. Preoperative cone beam computed tomography sections.
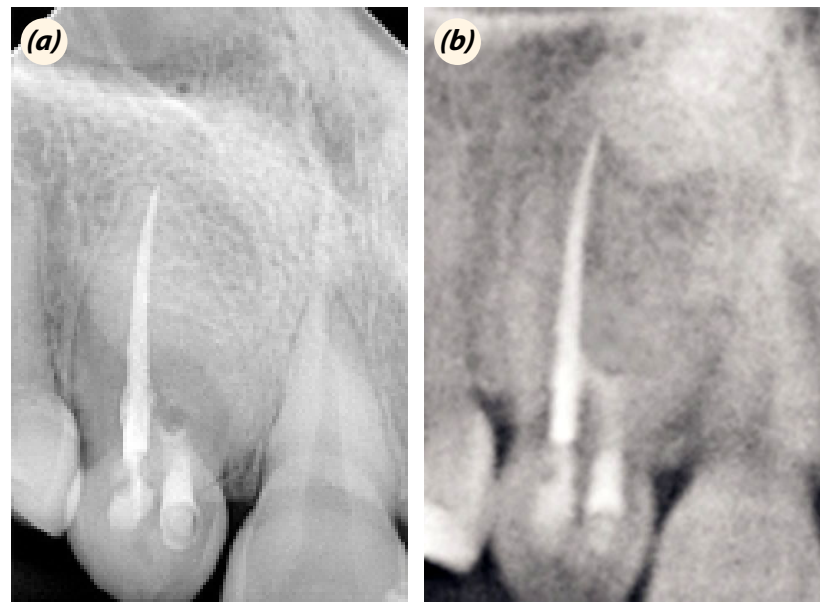

Fig. 4. (a) 6-months follow-up periapical radiography. (b) 1-year follow-up periapical radiography.

ionomer cement followed by composite resin over it (Luxacore; DMG, Hamburg, Germany).

The patient was followed up for 6-months, 1-year and 5 -year. At the re-call appointments, the patient was asymptomatic, without evidence of a sinus tract, and radiographic assessment demonstrated resolution of the radicular infection (Fig. 4a, b). Also, at the 6-months follow-up, on the tomography taken from the patient, new bone tissue formation and reduction at the lesion margins were detected. To make firm the lesion was healed, the 6-months tomography image was shown in Fig. 5 together with the

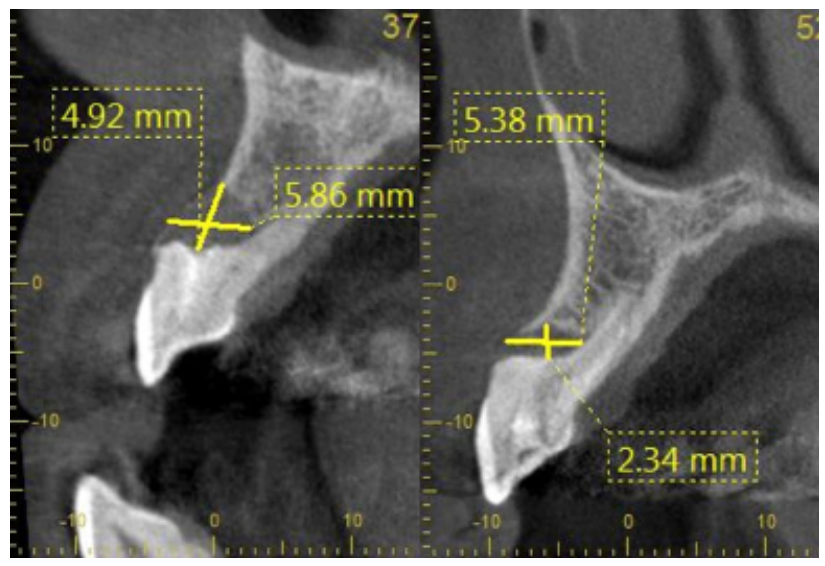

Fig. 5. Pre-op and 6-months follow-up tomography sections.

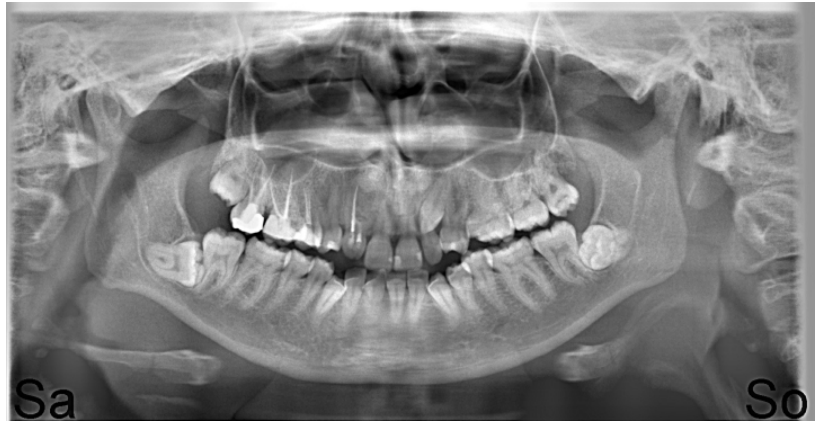

Fig. 6. 5-years follow-up panoramic radiography.

pre-op tomography section. After 5-year follow-up, radiographic assessment represented that the lesion was completely healed (Fig. 6). In the all follow-up appointments, the tooth was asymptomatic and functional.

\section{Discussion}

Dens invaginatus is obviously an endodontic challenge, mainly because of the complex root canal anatomy and the difficulty accessing the irregular and invaginated canals. ${ }^{[1]}$ In teeth with dens invaginatus, the invagination allows entry of irritants into the area that is separated from pulpal tissue only by a thin layer of enamel and dentine or some channels can make easy connection between the invagination and the pulp tissue. Therefore these teeth are vulnerable to early caries and pulp necrosis. ${ }^{[8,9]}$

In the most cases, dens invaginatus is detected by chance on the radiographs. Clinically, an abnormal crown shape (dilated, peg-shaped or barrel-shaped) or a deep foramen caecum may be important markers. ${ }^{[10]}$ In the present case, the crown was larger compared to the lateral incisor on the left side. However; affected teeth also can expose no clinical signs of the malformation. Therefore it generally progresses to pulpal necrosis without any characteristic symptoms. So early diagnosis and treatment of dens invaginatus is essential in order to prevent extra 
problems (abscess formation, retention of neighboring teeth,displacement of teeth, cysts, ${ }^{[11]}$ internal resorption and tooth missing.

Different ways are used to manage dens invaginatus, such as fissure sealant and conservative restorations at early stages, root canal therapy, and surgical endodontic treatment ${ }^{[12-14]}$ and extraction. ${ }^{[15]}$ Nonsurgical root canal treatment should be the first treatment choice before turning to endodontic surgery, intentional replantation, or extraction of the tooth. Surgical treatment is the second option and is only indicated in case of failure of the conservative root canal treatment, in teeth which cannot be treated nonsurgically because of anatomical variations or failure to gain access to all parts of the root canal system

Most endodontics prefer nonsurgical interventions (orthograde) toward the treatment of dens invaginatus. ${ }^{[16-21]}$ Pai et al. ${ }^{[18]}$ demonstrated that treatment of a tooth with type 3 dens invaginatus, immature apex, and an associated large periapical lesion is usually complicated. Nonsurgical endodontic therapy should be experienced it primarily.

Apexification with calcium hydroxide necessitates multiple appointments and also decreases the fracture strength of teeth. In recent times, considerable interest has been expressed in the use of other materials such as MTA. MTA can be considered an impeccable option in the endodontic therapy of teeth with immature apex with the advantage of shorter treatment time, good sealing ability, and high biocompatibility. It has been demonstrated that MTA induces the formation of a calcified matrix in the periapical tissue and regeneration of new cementum. ${ }^{[22,23]}$

In this case report, the case presented here was diagnosed as a rare and interesting variation Oehlers' tip III invaginatus with maxillary right lateral incisor. The related tooth has two root canals, a primary canal with normal anatomic form and an invaginated canal with necrotic tissue, which was associated with a periapical lesion around to apex of the tooth Successful conservative treatment of tooth without removing the anomaly has been managed. Many factors can affect the prognosis of nonsurgical endodontic treatment of this case. Firstly the extensive invaginated space was disinfected from microorganisms and enfecte tissue. Second the invagination was dressed with calcium hydroxide for a while to sterilize the infecte canal. Third, the widely open apex obturated with MTA because of apical sealing.

\section{Conclusion}

Based on the satisfactory outcomes of this case 5-year clinical and radiological follow-up; only nonsurgical end- odontic treatment must be the initial treatment choice before turning to other treatment options. Because this case report revealed that even type III dens invaginatus with an open apex and large periapical lesion, can be treated nonsurgically using MTA as an apical plug.

Conflict of interest: None declared.

Authorship contributionsः Concept: N.Y.Ç.; Design: E.Ç.; Supervision: N.Y.Ç., E.Ç.; Materials: N.Y.Ç., E.Ç.; Data: N.Y.Ç.; Analysis: N.Y.Ç., E.Ç.; Literature search: N.Y.Ç.; Writing: N.Y.Ç.; Critical revision: N.Y.Ç.

\section{References}

1. Alani A, Bishop K. Dens invaginatus. Part 1: classification, prevalence and aetiology. Int Endod J 2008;41:1123-36.

2. Hovland EJ, Block RM. Nonrecognition and subsequent endodontic treatment of dens invaginatus. J Endod 1977;3:360-2.

3. Hülsmann M. Dens invaginatus: aetiology, classification, prevalence, diagnosis, and treatment considerations. Int Endod J 1997;30:79-90.

4. Hosey MT, Bedi R. Multiple dens invaginatus in two brothers. Endod Dent Traumatol 1996;12:44-7.

5. Oehlers FA. Dens invaginatus (dilated composite odontome). I. Variations of the invagination process and associated anterior crown forms. Oral Surg Oral Med Oral Pathol 1957;10:1204-18

6. Gonçalves A, Gonçalves M, Oliveira DP, Gonçalves N. Dens invaginatus type III: report of a case and 10-year radiographic follow-up. Int Endod J 2002;35:873-9.

7. Wong M. Treatment considerations in a geminated maxillary lateral incisor. J Endod 1991;17:179-81.

8. Heydari A, Rahmani M. Treatment of Dens Invagination in a Maxillary Lateral Incisor: A Case Report. Iran Endod J 2015;10:207-9.

9. Nik-Hussein NN. Dens invaginatus: complications and treatment of non-vital infected tooth. J Clin Pediatr Dent 1994;18:303-6.

10. Subbiya A, Geethapriya N, Pradeepkumar AR, Vivekanandhan P. An Unusual Type II Dens Invaginatus with Calcified Canals in a Maxillary Lateral Incisor - A Case Report. J Clin Diagn Res 2013;7:2084-5.

11. Conklin WW. Bilateral dens invaginatus in the mandibular incisor region. Oral Surg Oral Med Oral Pathol 1978;45:905-8.

12. Hata G, Toda T. Treatment of dens invaginatus by endodontic therapy, apicocurettage, and retrofilling. J Endod 1987;13:469-72.

13. Suchina JA, Ludington JR Jr, Madden RM. Dens invaginatus of a maxillary lateral incisor: endodontic treatment. Oral Surg Oral Med Oral Pathol 1989;68:467-71.

14. Kulild JC, Weller RN. Treatment considerations in dens 
invaginatus. J Endod 1989;15:381-4.

15. De Smit A, Demaut L. Nonsurgical endodontic treatment of invaginated teeth. J Endod 1982;8:506-11.

16. Castelo-Baz P, Martın-Biedma B, Varela-Patino P. Combined endodontic and surgical managementof dens invaginatus in a central maxillary incisor diagnosed by cone beam computed tomography: case report and 2-year follow-up. ENDO (Lond Engl) 2014;8:157-62.

17. Sübay RK, Kayataş M. Dens invaginatus in an immature maxillary lateral incisor: a case report of complex endodontic treatment. Oral Surg Oral Med Oral Pathol Oral Radiol Endod 2006;102:e37-41.

18. Pai SF, Yang SF, Lin LM. Nonsurgical endodontic treatment of dens invaginatus with large periradicular lesion: a case report. J Endod 2004;30:597-600.

19. Steffen H, Splieth C. Conventional treatment of dens invaginatus in maxillary lateral incisor with sinus tract: one year follow-up. J Endod 2005;31:130-3.
20. Narayana P, Hartwell GR, Wallace R, Nair UP. Endodontic clinical management of a dens invaginatus case by using a unique treatment approach: a case report. J Endod 2012;38:1145-8.

21. Kumar H, Al-Ali M, Parashos P, Manton DJ. Management of 2 teeth diagnosed with dens invaginatus with regenerative endodontics and apexification in the same patient: a case report and review. J Endod 2014;40:725-31.

22. Agrawal PK, Wankhade J, Warhadpande M. A Rare Case of Type III Dens Invaginatus in a Mandibular Second Premolar and Its Nonsurgical Endodontic Management by Using Cone-beam Computed Tomography: A Case Report. J Endod 2016;42:669-72.

23. de Oliveira Paulo A, Tanomaru-Filho $M$, de Toledo Leonardo R, Chavez-Andrade GM,Guerreiro-Tanoma$\mathrm{ru}$ JM Apexification with white MTA in an immature permanent tooth with dens invaginatus. Braz J Oral Sci $2013 ; 12: 61-5$. 